\section{Molecular immunology}

Immunochemistry: An Advanced Textbook. Edited by L. E. Glynn and M. W. Steward. Pp. 628. (Wiley: New York and Chichester, 1977.) $£ 24$.

RECENT years have seen the publication of several excellent advanced texts in cellular immunology and in practical methods in immunology. The immunochemist, or indeed anyone interested in reading about general molecular immunology at a level beyond undergraduate textbooks, has not been so well served and has had to rely on out-dated books or on isolated reviews. This volume is therefore a particularly timely and welcome publication. It is a collection of reviews covering the structure, function, genetics and biosynthesis of antibodies, their reactions with antigens (in general and in certain selected cases) and the complement system.

I particularly appreciated the chapter by Stanworth on immunoglobulinopathies. Easily understandable to the non-clinician, this chapter presents the clinical background to multiple myeloma and other immunological disorders that underlies so much of our knowledge of antibody structure and function but is often absent from the non-clinical ilterature. A short chapter on amyloid (Pras and Gafni) also bridges the clinical and non-clinical aspects of the subject. The chapters on three-dimensional immunoglobulin structure (Feinstein and Beale; Richards et al.) are extremely good non-mathematical accounts of crystallographic and physicochemical techniques and results. Thermodynamics and kinetics of antibody-antigen reactions are also presented in a very readable explanation of the factors relating to antibody affinity and activity (Steward).

Williamson, in a chapter on the origin of antibody diversity discusses evidence relating to, and some models that have been proposed to account for the enormous diversity of antibodies. He concludes that a germ-line basis for antibody diversity best explains the available data. It is unfortunate that this view is not balanced by an equally forceful presentation of the case for the somatic generation of antibody diversity elsewhere in the book.

The chapter on complement (Fearon and Austen) is a straightforward account of the components and reactions of the complement systems. I should have liked to have seen more discussion of the genetic association between certain complement components and the major histocompatibility system. Space prevents me from men- tioning all sixteen chapters, but one other section that I found very informative was that on structure-function relationships of immunoglobulins (Turner).

The length, degree of complexity and flavour of each chapter varies widely according to the specialist authors chosen to contribute in their field. The style adopted ranges from a straightforward summary of data in some chapters to a more speculative, and even controversial, discussion in others. However, each chapter is followed by an extensive list of references (up to 1975 or early 1976), allowing the more serious student to sample the original literature. There is also what seems to be a fairly comprehensive index, though my only genine attempt to use it - to find the reference to Forssmann, (or is it Forsmann, as both spellings are used in this book) antibody, given as p383-was unsuccessful. Cross-referencing between chapters could also have been made easier by indicating the chapter number on each page (or at the beginning of each section).

My main criticism of the book, how-

\section{Epithelial physiology and ion transport processes}

Membrane Transport Processes. Vol. 1. Edited by J. F. Hoffman. Pp.476. Vol. 2. Edited by Daniel C. Tosteson, Yu. V. Ovchinnikov and Ramon Latorre. Pp.451. (Raven: New York, 1978.) $\$ 42$ each volume.

Ir was with some trepidation that I agreed to review these two volumes. All too often these days the ephemera of meetings are presented to us encased in the respectability of hard covers and my hope was that these volumes would not prove to be just another example of this publishers' ploy. As I began to read, it became clear that at least for volume 1 my fears were groundless.

The first volume arose out of a small meeting held in memory of Peter Curran. To the papers given on that occasion, others have been added to produce a very useful survey of epithelial physiology and closely related topics. Almost without exception the chapters are authoritative and scholarly contributions of lasting value -a fitting tribute to Peter Curran, whose ideas and work in this field were so seminal. The names of most of the best known American epitheliologists are to be found amongst the contributors; but it is perhaps a pity that the rest of the world is rather poorly rep- ever, arises from its claim to be a textbook. The variety of authors' stylesone of the attractions of the volume as a collection of reviews-is not well suited to a textbook. Nor is the overlap of data (and even photographs) between chapters; and the numerous minor errors that, in the interests of topicality, one might excuse in a review as a consequence of hurried proofreading, should not be acceptable in a textbook.

But whatever one calls it, I enjoyed reading this volume enormously. My copy is already well-thumbed, but I shall enjoy re-reading many of the chapters and will also frequently refer to it for information. It is most unfortunate that the publishers seem to be resigned to library sales only-at $£ 24$ I cannot see the book becoming an immunologist's vade mecum, but it would nevertheless be a useful addition to any immunology library.

David Secher

David Sechar is a member of the staff of the MRC Laboratory of Molecular Biology, Cambridge, UK.

resented. One might have hoped for a chapter on the recent exciting contributions of electron microprobe X-ray analysis, and it is unfortunate that the editor has allowed Diamond to dismiss the work of Hill in a series of asides when Hill could have been invited to put his own case. I am sure that Peter Curran would have wished Hill's views -right or wrong-to have been given a less biassed hearing.

These criticisms are, however, of a very minor nature and should not be allowed to detract from an excellent volume. The editor and authors are to be congratulated on producing a valuable work of scholarship that will be much used in years to come.

Try as I must, I cannot say the same about volume 2 , which covers a very broad range of topics concerned with the selective transport of ions across biological membranes. Here the main selling point seems to be a number of contributions from our Russian colleagues. The volume is very mixed, with some excellent articles-for instance, Kostyuk and Krishtal on ionic and gating mechanisms in molluscan neurones; but also some very poor ones. It lacks the consistent scholarly solidarity of volume 1 and it is difficult to understand for what purpose this particular pot pourri of papers was put together. Regretfully, I cannot place this volume in even the same league as its sister.

\section{P. F. Baker}

P. F. Baker is Professor of Physiology at King's College, University of London, UR, 